\title{
Relative judgment in facial identity perception as revealed by sequential effects
}

\author{
Shen-Mou Hsu ${ }^{1}$ • Jung-Sen Lee ${ }^{1}$ \\ Published online: 10 September 2015 \\ (C) The Psychonomic Society, Inc. 2015
}

\begin{abstract}
Existing models of facial identity perception often assume that information conveyed by facial stimuli provides the sole basis for identity judgments, largely ignoring the involvement of contextual effects. Capitalizing on sequential effects, the present study investigates whether facial identity is judged relative to a context shaped by stimuli presented in previous trials. When categorizing a sequence of facial identities, our results demonstrated that participants' categorization of current faces varied according to the local sequential context provided by the immediately preceding faces and, to some extent, by the preceding stimuli presented two trials prior to the current trial. Moreover, this variation depended on the relative distance between the preceding and current faces. Notably, the nature of these identity-based sequential effects was qualitatively different between male and female participants. Female participants tended to respond to the current faces with the same category label as on the preceding faces. However, male participants responded with the same label only when the relative distance was small, but responded with a different label when the relative distance was increasingly large. The present study demonstrates that relative information between the preceding and current faces may be used as evidence to inform a judgment. However, this process is multifaceted rather than unitary and depends in part on participant gender.
\end{abstract}

Shen-Mou Hsu

smhsu@nccu.edu.tw

1 Research Center for Mind, Brain and Learning, National Chengchi University, NO.64, Sec.2, ZhiNan Rd., Wenshan District, Taipei 11605, Taiwan, Republic of China
Keywords Assimilation · Contrast · Face · Identity · Sequential effects

\section{Introduction}

The ability to identify an individual's face represents one of the highlights of the human cognitive system. Given that all faces have the same basic configural appearance, representations of facial identity must be sufficiently robust to distinguish subtle variations in the facial characteristics of individuals. Existing models of facial identity perception frequently assume that there is direct mapping between stimulus features and corresponding perceptual judgments (Bruce \& Young, 1986; Valentine, 1991). Thus, the information conveyed by facial stimuli may serve as the sole basis for judging facial identity. For example, according to the influential face space metaphor (Valentine, 1991), individual faces are conceptualized as distinct locations in a multidimensional space, and the dimensions of this space correspond to various aspects of the facial characteristics used for individuation. The location information in this space represents the absolute features used for the perceptual judgments of facial identity. However, relative judgment also is widely used in psychophysical (Helson, 1964; Laming, 1997; Lockhead, 2004) and social (Mussweiler, 2003) judgments. It has been posited that when a stimulus is presented, a perceptual judgment is rendered via comparison to an implicit standard, and one important source of the standard is a context shaped by previous material. For example, in the magnitude estimation of tones of varying loudness (Ward \& Lockhead, 1970), a neutral tone is judged as louder than it actually is if preceded by a loud tone, but the same tone is judged as quieter than it actually is if preceded by a quiet tone. 
The present study investigated whether the information provided by current facial stimuli determines the judgments of facial identity or if current facial identity is judged relative to preceding contextual information. We probed this issue by using sequential effects. Recently, there has been a resurgence of interest in sequential effects, particularly during binary categorization processing (Hampton, Estes, \& Simmons, 2005; Stewart, Brown, \& Chater, 2002; Zotov, Jones, \& Mewhort, 2011). In these studies, when a sequence of stimuli is presented, the categorization responses to the stimuli in current trials have been found to vary according to the local sequential context shaped by the immediately preceding stimuli presented one trial back. Although the presence of such sequential effects indicates that a current judgment is made relative to preceding contexts, interpretations regarding the nature of these phenomena remain inconclusive. One interpretation suggests that sequential effects involve the computation of similarity/dissimilarity between preceding and current stimuli to guide perceptual judgments (Stewart \& Brown, 2005; Stewart, et al., 2002). An alternative interpretation argues that sequential effects reflect the consequences regarding how the local sequential context shifts participants' internal criteria to the current category representation (Hampton, et al., 2005; Zotov, et al., 2011).

Despite this disagreement, two types of bias have frequently been observed in sequential effects during categorization, depending on the relative distance (i.e., similarity) between the preceding and current stimuli. One bias is contrast effects: When the relative distance between the current and preceding stimuli is large, the current stimuli are categorized as further from the category of the preceding stimuli than they actually are. For example, when participants learn to categorize a continuum of 10 equally spaced tones, with the 5 lowest frequency tones in Category A (tones 1 to 5) and the 5 highest frequency tones in Category B (tones 6 to 10), participants are more likely to categorize tone 5 as "Category A" when it follows distant tone 10 from Category B than when it follows distant tone 1 from Category A (Stewart, et al., 2002). This bias occurs because participants contrast their judgments away from the category of a distant preceding stimulus, which leads to more correct responses if preceded by a stimulus from the opposite category but to more errors if preceded by a stimulus from the same category.

Conversely, assimilation effects indicate that current stimuli are categorized as closer to the category of the preceding stimuli than they actually are when the interstimulus distance is small. For example, when a sequence of facial expressions is categorized in such a manner that the physical features of the stimuli are morphed continuously between two categories of emotion (e.g., fear and disgust), participants are more likely to categorize a morphed fearful expression as "fearful" when it is preceded by a nearby fearful morph as opposed to a nearby disgusted morph
(Hsu \& Yang, 2013). This bias occurs because participants assimilate their judgments toward the category of a nearby preceding stimulus, which leads to more correct responses if preceded by a stimulus from the same category but to more errors if preceded by a stimulus from the opposite category.

In the present study, participants performed a similar binary categorization task on a sequence of facial identities that comprised a continuum of the morphed faces of two celebrities. If judgments of facial identity are dependent on local sequential contexts, we expect that the responses to the same facial stimuli will differ according to different local sequential contexts provided by immediately preceding facial stimuli. Nevertheless, perceptual judgments of facial identity are further complicated by the genders of the faces. Prior studies have shown that the processes of facial gender and facial identity may mutually interact. For example, using a Garner interference task (Ganel \& Goshen-Gottstein, 2002), reaction time performance in gender classification was found to be impeded by irrelevant variations in facial identities. Reciprocally, irrelevant variations in facial gender also could interfere with identity classification. Capitalizing on facial identity aftereffects, evidence (Rhodes, et al., 2011) has suggested that facial gender determines how facial identities are represented, with male and female faces encoded along dissociable dimensions within the facial identity space. In addition to facial gender, participants' gender differences in the perceptual judgments of facial identity have been reported (Herlitz \& Loven, 2013; Lewin \& Herlitz, 2002; Wright \& Sladden, 2003). Females are better at recognizing female faces; however, the tendency for males to be better at recognizing male faces is less clear. Altogether, the goal of the present study is twofold: 1) we examine the involvement of relative judgment in facial identity perception in the context of sequential effects between the current and immediately preceding faces, and 2) we further explore whether potential identity-based sequential effects are exhibited in the same manner across participants and faces of different genders and whether these effects involve contrast or assimilation effects.

\section{Methods}

\section{Participants}

Fourteen right-handed males (mean age $=22.23$ years, range $=$ 20-26) and 14 right-handed females (mean age $=22.21$ years, range $=19-29)$ participated in the experiment. All participants had normal or corrected-to-normal vision and provided written, informed consent prior to the study. 


\section{Stimuli}

The original stimuli comprised 16 portraits of celebrities $(8$ males and 8 females) collected from the Internet. To optimize performance, the participants first provided familiarity ratings for all celebrities without viewing the corresponding stimuli. The participants indicated how well they knew that celebrity on a scale of 1 to $3: 1$ ) "I have not heard of this celebrity at all"; 2) "I have heard of this celebrity but am not familiar with the face"; or 3) "I have heard of this celebrity and am familiar with the face." Six male and six female celebrities were then selected for each participant according to the participants' rating scores (all selected faces had a score of 3 ). As a result of this screening process, the content of the selected facial stimuli differed among the individuals.

The appropriate faces were divided into six gendermatched pairs ( 3 male pairs and 3 female pairs) based on whether the paired faces had a similar composition and facial expression. The faces were subsequently subjected to a morphing procedure to create six continua of morphed facial identities using FantaMorph (Abrosoft). In each face identity continuum, celebrity A's face was morphed $10 \%, 20 \%, 30 \%$, $40 \%, 50 \%, 60 \%, 70 \%, 80 \%$, and $90 \%$ of the physical distance to paired celebrity B's face (Fig. 1, bottom panel). Moreover, the stimuli in each continuum were adjusted and matched on low-level physical attributes (luminance, contrast, and spatial frequency) using the SHINE toolbox (Willenbockel, et al., 2010). The face images subtended a horizontal visual angle of $6.8^{\circ}$ and a vertical angle of $8.6^{\circ}$ around the center of the screen, with a viewing distance of approximately $60 \mathrm{~cm}$.

\section{Procedure}

Because there were six identity continua ( 3 males and 3 females), the participants had to complete six randomized blocks of trials, with one block for each continuum. There were breaks between blocks. Each block comprised two runs. Within each run, all facial stimuli along a facial-identity continuum were repeated 9 times, which resulted in 99 randomly presented trials $(9$ repetitions $\times 11$ faces per continuum).

A trial was initiated with a fixation cross at the screen center for $600 \mathrm{~ms}$. A facial stimulus was randomly selected from a given facial continuum and presented for $400 \mathrm{~ms}$. A response window was subsequently displayed with two choices that indicated the two identities used to create the continuum. The response choices were placed on either side of the fixation cross, and the positions of these choices were randomized across trials. The participants were given up to $3 \mathrm{~s}$ to classify the face they had just viewed by pressing a corresponding key. Performance feedback was not provided. The key press initiated a new trial after 500-ms intertrial interval.

\section{Results}

\section{Categorization data of identity continua}

For each continuum, the categorization data of each morphed face were calculated as the proportion of choices that corresponded to the two identity categories used to create the continuum. The responses to stimuli at identical morph steps were first averaged, regardless of their sequential contexts. Figure $1 \mathrm{a}$ and $\mathrm{b}$, respectively, show the data from one representative continuum from a single participant or collapsed across participants. A highly consistent picture emerged for each continuum. Every continuum roughly contained two regions that were separated by a category boundary with a categorization rate of approximately $50 \%$ (mean categorization rate $\pm S E M=50.86 \pm 0.90 \%$, collapsed across identity categories, continua and participants). In addition, each region belonged to the identity category that corresponded to the prototype at that end of the continuum. Notably, depending on the continua and the individuals, the exact location of the category boundaries varied between 70:30 and 30:70 morphs.

\section{Stimulus selection}

To control for the variability in category boundaries to properly examine the effects of sequential contexts, our analyses first focused on the two highly recognizable target morphs that were closest to the category boundaries for each continuum (one morph in each identity category) because this is the region in which the strongest sequential effects were previously observed (Hampton, et al., 2005; Hsu \& Yang, 2013; Stewart, et al., 2002). Moreover, for every continuum, we analyzed how categorization responses to the targets varied when the targets were preceded by the following preceding stimulus types in each identity category: the prototypes at the ends of the continuum (Pfaces), the targets themselves and the morphs (M-faces) at the mid-points of the P-faces and targets (if the exact mid points did not exist, we selected the morphs around the potential mid points but closer to the targets as the Mfaces). The gray bars in Fig. 1a illustrate an example of how these faces were selected for one representative continuum from a single participant. All these selected faces were highly recognizable and judged as belonging to a distinct identity category with categorization rates at or greater than $77.78 \%$ for any one continuum and participant. After being collapsed across identity categories, continua and participants, the average categorization rates for these selected faces were as follows: P-face, mean categorization rate $\pm S E M=97.94 \pm 0.48 \%$; M-face, $96.93 \pm 0.62 \%$; target, $88.30 \pm 0.46 \%$. 
Fig. 1 Categorization data for the facial-identity continuum. Data were obtained from one representative continuum from a single participant (a) or after being collapsed across the participants (b). The continuum ranges from the prototype of celebrity A to the prototype of celebrity B in 10 morphing steps. It should be noted that depending on the continua and participants, the exact location of a category boundary is between $70: 30$ and 30:70 morphs. The gray bars indicate the expression stimuli selected to analyze sequential effects. The notations $\mathrm{P}$ and $\mathrm{M}$ represent the preceding $\mathrm{P}$-faces and $\mathrm{M}$-faces, respectively. Error bars represent \pm SEM

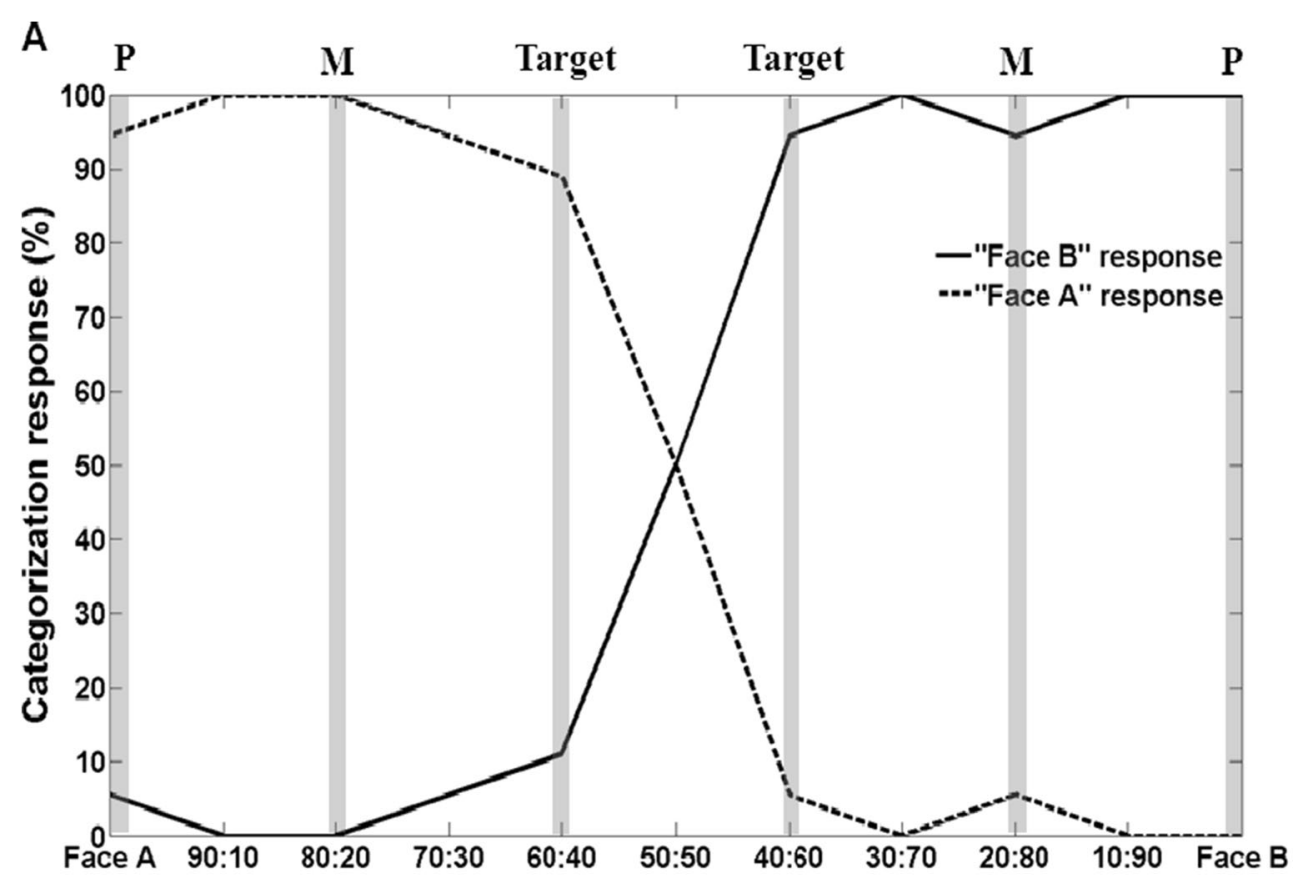

B

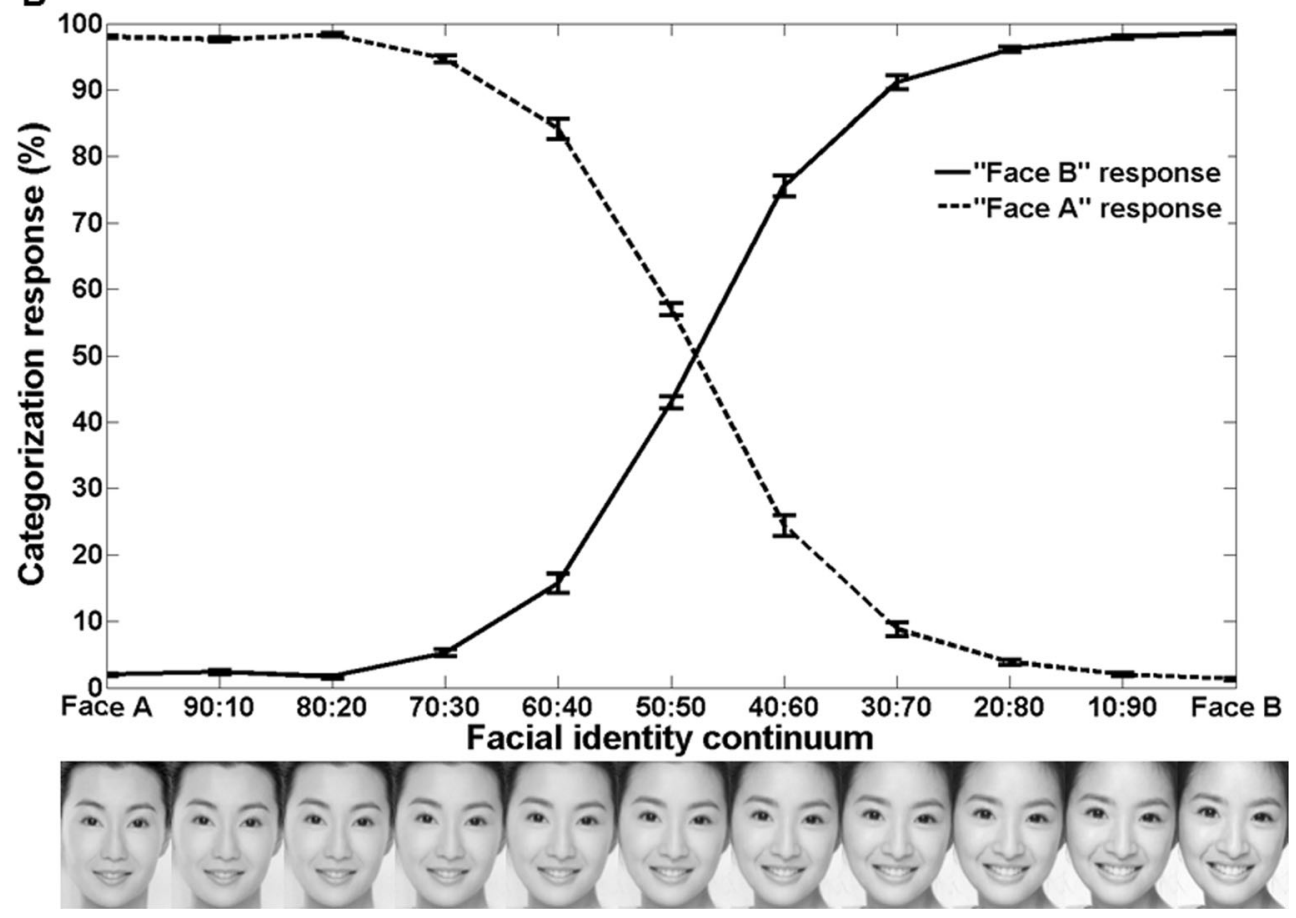

\section{General sequential effects}

To investigate the respective roles of facial and participant gender, modal ("accurate") categorization responses were calculated separately for male and female targets and for male and female participants. Modal responses were defined as the dominant or the majority category choices that participants assigned to the face stimuli. In each condition (Fig. 2a-d), responses to the two targets within the same continuum were first analyzed as a function of the preceding stimuli and then pooled together. Next, the obtained results were collapsed across continua. The preceding stimuli were the previously selected P-faces, M-faces, and targets, depending on whether these were of the same category as the current targets (white zone) or different identity categories from the current targets (gray zone). Notably, preceding stimuli and targets had the same facial gender, because the continua were gendermatched. In addition, we did not analyze trials in which the targets were preceded by the stimuli whose responses were non-modal ("inaccurate") to ensure that differential 
A Male participant -Male target

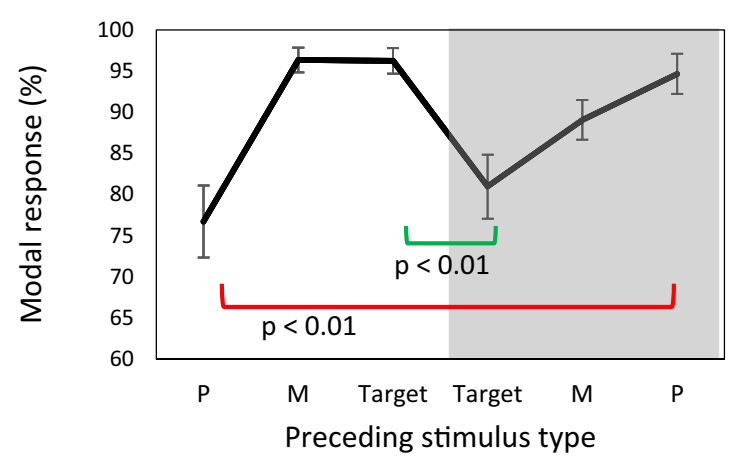

C Female participant -Male target

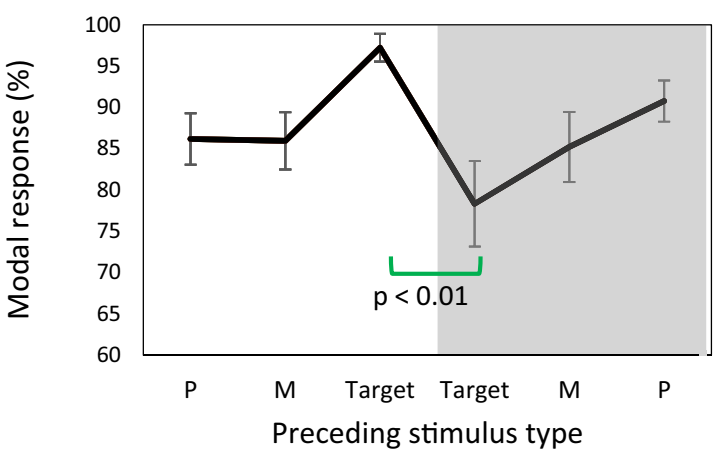

Fig. 2 Modal responses to the current facial targets as a function of the preceding stimulus types when male participants categorized male (a) and female (b) targets and female participants categorized male (c) and female (d) targets. The gray zones indicate that the preceding and current facial stimuli have different category memberships. Error bars represent \pm SEM.

categorization rates among the preceding P-, M-, and target faces would not contaminate the results.

The overall data collapsed across facial and participant genders were first analyzed. The results demonstrated the presence of sequential effects in facial identity perception, as categorization of current facial identities varied as a function of preceding stimulus types $(2 \times 2 \times 6$ mixed ANOVA, main effect of preceding stimulus type, $F(5,130)=20.93, p<$ $0.001)$. Although the modal responses to the male and female targets were comparable (main effect of facial gender, $F(1,26)$ $=0.17, p=0.681$ ), responses did significantly differ between male and female participants (main effect of participant gender, $F(1,26)=10.66, p=0.003)$. Further analyses did not reveal any significant three-way interaction effect $(F(5,130)$ $=1.99, p=0.099$ ), nor were there significant two-way interaction effects between preceding stimulus type and facial gen$\operatorname{der}(F(5,130)=0.88, p=0.496)$ or between facial and participant gender $(F(1,130)=1.85, p=0.237)$. Significant interaction was found between preceding stimulus type and participant gender $(F(5,130)=2.38, p=0.042)$, indicating that the patterns of sequential effects exhibited differently between male and female participants.
B Male participant -Female target

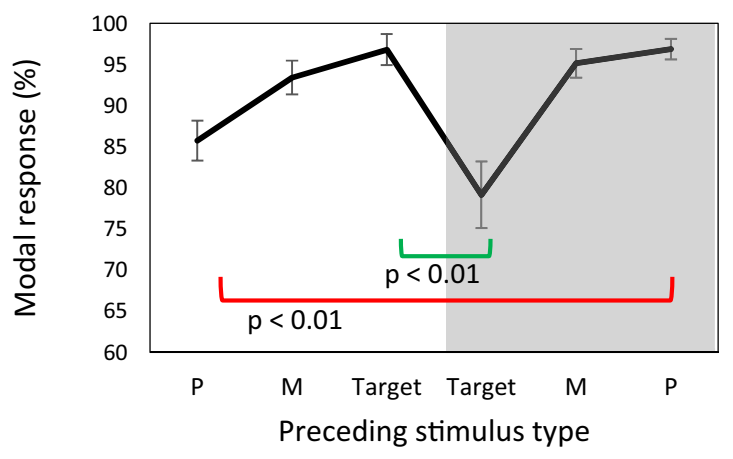

D Female participant -Female target

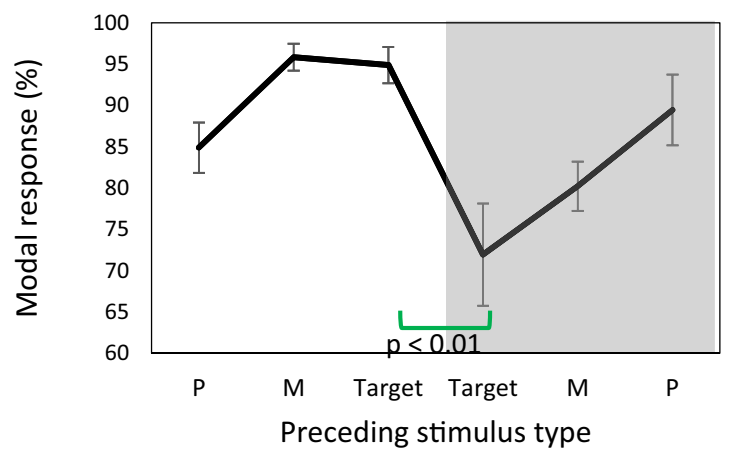

After being collapsed across facial and participant genders, the average number of trials used to compute the data for each preceding stimulus type are as follows. P, $9.71 \pm 0.74 ; \mathrm{M}, 9.61 \pm 0.87$; Target, $8.16 \pm 0.78$; Target (gray zone), $7.73 \pm 0.86 ; \mathrm{M}$ (gray zone), $9.43 \pm 0.85 ; \mathrm{P}$ (gray zone), $9.64 \pm 0.89$

Next, we separately examined the presence of identitybased sequential effects according to facial and participant gender. For male participants, the results showed that the categorization of both current male and current female targets differed significantly according to the preceding stimulus types (male targets: one-way repeated-measures ANOVA, $F(5,65)=8.57, p<0.001$, Fig. 2a; female targets: $F(5,65)$ $=9.12, p<0.001$, Fig. 2b). Similarly, for female participants, the modal responses differed for male targets $(F(3,39)=6.57$, $p<0.001$, Fig. 2c) as well as for female targets $(F(5,65)=$ $3.40, p=0.009$, Fig. $2 \mathrm{~d}$ ). In general, these findings provide supporting evidence that the categorical judgments of current facial identities were affected by the local sequential context provided by the immediately preceding stimuli.

\section{Effect of relative distance during same- and different-category transitions}

To further understand the nature of the obtained sequential effects, we assessed how the effects depended on the relative distance between the preceding and current stimuli and whether this dependence differed when the preceding and current 
stimuli had the same or different identity category memberships. To this end, trends analyses were conducted to qualitatively estimate whether the relative distance between two successive faces influenced sequential effects in a linear fashion under each condition.

For male participants, when the preceding and current faces originated from the same identity category (same-category transitions), the modal categorization judgments of current male targets were increased, with decreasing relative distance between the preceding and current faces $(F(1,13)=17.28, p=$ 0.001 , the white zone in Fig. 2a). This linear trend also was observed for the judgments of current female faces $(F(1,13)=$ $7.17, p=0.003$, the white zone in Fig. 2b). Conversely, when the preceding and current stimuli originated from different identity categories (different-category transitions), the modal responses to current male $(F(1,13)=13.28, p=0.003$, the gray zone in Fig. 2a) and female targets $(F(1,13)=20.93, p=$ 0.001 , the gray zone in Fig. 2b) were increased, with increasing relative distance between the preceding and current faces.

For female participants, a similar pattern of results was obtained. During the same-category transitions, they performed better with decreasing relative distance between the preceding and current male faces $(F(1,13)=10.73, p=0.006$, the white zone in Fig. 2c) and between the preceding and current female faces $(F(1,13)=13.28, p=0.003$, the white zone in Fig. 2d). Conversely, during the different-category transitions, categorization responses were increased for current male $(F(1,13)=4.76, p=0.048$, the gray zone in Fig. $2 c)$ and female faces $(F(1,13)=8.08, p=0.014$, the gray zone in Fig. 2d), with increasing relative distance between the two successive stimuli.

In summary, the relative distance between the preceding and current faces plays a crucial role in determining the sequential effects. Moreover, the same pattern of relativedistance effects was observed in both male and female participants. On the one hand, during the same-category transitions, an increased inter-stimulus distance was accompanied by reduced modal responses for the current targets. On the other hand, during the different-category transitions, an increased interstimulus distance was associated with enhanced modal responses for the current targets. This pattern of findings can be explained by contrast effects. From this perspective, the participants may be biased in categorizing the current faces as away from the category of the preceding faces when the inter-stimulus distance is increasingly large. This bias yielded decreased modal responses for the current targets following more distant faces from the same category or increased modal responses following more distant faces from the opposite category. However, a complementary indication is that the identical findings reflect assimilation effects in which the targets are judged as close to the category of the preceding faces when the interstimulus distance is increasingly small. Consequently, the modal responses to the current targets were increased after viewing more nearby preceding faces from the same category or fewer modal responses were induced after viewing more nearby preceding faces from the opposite category.

\section{Contrast and assimilation effects}

We proceeded to examine whether our findings could best be construed as reflecting contrast, assimilation effects or both. As reported in previous research (Stewart, et al., 2002), contrast effects may be operationally defined by examining the responses to the current targets after viewing a distant stimulus from the opposite category relative to a distant stimulus from the same category. This definition is based on that if participants classify current stimuli as further from the category of a distant preceding stimulus, they would produce more modal ("correct") responses when the current stimulus is preceded by a stimulus from the opposite category but produce fewer modal responses ("more errors") when the current stimulus is preceded by a stimulus from the same category. Such a switch in categorization responses may thus optimize the phenomena of contrast effects.

For male participants, we did find increased modal responses in both male and female targets when the preceding stimuli were the distant P-faces from the opposite category relative to the distant $\mathrm{P}$-faces from the same category (male targets: paired $t$-test, $t(13)=3.41, p=0.005$, red-solid bracket in Fig. 2a; female targets: $t(13)=3.84, p=0.002$, red-solid bracket in Fig. 2b), which indicates the involvement of contrast effects. However, no contrast effect was found for female participants when they categorized either male targets $(t(13)=$ $1.13, p=0.278$, Fig. $2 \mathrm{c})$ or female targets $(t(13)=0.82, p=$ 0.429 , Fig. $2 \mathrm{~d}$ ). When categorizing male facial targets, a separate analysis confirmed that the observed contrast effect was specific in male participants, because the size of the effect was stronger in males than in females (two-sample $t$-test, $t(13)=$ $2.01, p=0.05)$. However, for female targets, the size was not significantly different according to participant gender $(t(13)=$ $1.04, p=0.31$ ).

Conversely, assimilation effects may be operationally defined by examining the responses to the current targets after viewing a nearby stimulus from the same category relative to after viewing a nearby stimulus from the opposite category. Because participants now classify current stimuli as close to the category of a nearby preceding stimulus when the relative distance between successive stimuli is small, this bias may lead to increased modal responses (higher "accuracy") if preceded by a stimulus from the same category but decreased modal responses (reduced "accuracy") if preceded by a stimulus from the opposite category.

Evidence of assimilation effects was found for the female participants when they categorized the current male and female targets because there was an increased modal responses in categorizing the targets after viewing the same target faces 
from the same category than after viewing the nearby target faces from the opposite category (male targets: $t(13)=3.50, p$ $=0.004$, green-solid bracket Fig. $2 \mathrm{c}$; female targets: $t(13)=$ $3.44, p=0.004$, green-solid bracket in Fig. 2d). Assimilation effects also were observed when male participants categorized current targets of both genders (male targets: $t(13)=3.50, p=$ 0.004, green-solid bracket in Fig. 2a; female targets: $t(13)=$ $3.86, p=0.002$, green-solid bracket in Fig. 2b). A separate two-sample $t$-test showed that, for both male and female targets, the sizes of the assimilation effects were comparable between male and female participants (male target: $t(13)=$ $0.52, p=0.607$; female target: $t(13)=0.66, p=0.518)$, indicating that the assimilation effect was not specific to participant gender.

Additional analysis revealed that assimilation and contrast effects could still be obtained in the overall data (assimilation effect: $t(13)=7.01, p<0.001$; contrast effect: $t(13)=4.44, p<$ 0.001 ). Our results thus indicate that these two effects are differentially involved in the performance of male and female participants. These results also closely agree with our previous ANOVA analysis which shows an interaction effect between preceding contexts and participant gender.

\section{Long-term sequential effects}

Were identity-based sequential effects limited to the influences from the immediately preceding stimuli or whether the stimuli presented earlier in the trial sequence, such as two trials back, still had an impact on the categorization responses to current facial identities? As shown in Fig. 3, the sequential effects derived from the preceding faces presented two trials back were still present, as the categorization of current facial identities varied according to the preceding stimulus types (2 $\times 2 \times 6$ mixed ANOVA, main effect of preceding stimulus type, $F(5,130)=6.75, p<0.001)$. There were no main effects of facial gender, participant gender and interaction effects (all $p s>0.319)$. Further analysis revealed that this long-term sequential effect was driven by the female participants (male targets: one-way repeated-measures ANOVA, $F(5,65)=$ $2.80, p=0.024$, Fig. 3 c; female targets: $F(5,65)=3.08, p=$ 0.015 , Fig. $3 \mathrm{~d}$ ) and not by the male participants (male targets: $F(5,65)=1.42, p=0.229$, Fig. 3a; female targets: $F(5,65)=$ $1.60, p=0.174$, Fig. $3 b$ ). In female participants, the effects varied in a significant linear manner with increasing relative distance between the female preceding and current faces during the different-category transitions (trend analysis, $\mathrm{F}(1,13)$ $=3.36, p=0.013$, the gray zone in Fig. 3d), but the influence of relative distance was not found in all the other conditions (all ps $>0.106$ ). Moreover, female participants still exhibited a tendency to assimilate their current responses to the category of the preceding stimuli presented two trials back (i.e., assimilation effect) when they judged both male $(t(13)=2.60, p=$ 0.022 , Fig. 3c) and female current faces $(t(13)=3.42, p=$
0.005, Fig. 3d). Together, long-term sequential effects were observed only in female participants under certain conditions, indicating that these effects are reduced when there is a twotrial gap between the preceding and current facial identities. It should be noted that the data points in some participants were based on as few as five trials.

\section{Control analysis}

Given that the exact stimulus content varied among participants (see "Stimuli" section), could this variance explain the participants' gender effect? To address this issue, we first ensured that the patterns of categorization perception data, i.e., categorization performance along each continuum, were comparable across continua and participants of different genders (Pearson correlation: $r>0.997$, all $p$ values $<0.001$ ). Second, we examined whether the nature of the selected stimuli was consistent regardless of the gender of the faces and participants. To this end, the mean categorization rates of the P-faces, B-faces, and targets in male or female facial continua were separately calculated for male and female participants. As shown in Fig. 4, we did not observe any significant difference in categorization between male and female P-faces (two-way mixed ANOVA, main effect on facial gender, $F(1,26)=3.91$, $p=0.06)$, male and female $\mathrm{M}$-faces $(F(1,26)=0.36, p=$ $0.55)$, or male and female targets $(F(1,26)=2.15, p=0.16)$. In addition, female and male participants performed comparably on all three types of faces (P-face: main effect on participant gender, $F(1,26)=0.25, p=0.62$; M-face: $F(1,26)=$ $2.97, p=0.10$; target: $F(1,26)=0.88, p=0.36$ ). Finally, there was no significant face-gender $\times$ gender-group interaction effect (P-face: $F(1,26)=0.68, p=0.42 ; \mathrm{M}$-face: $F(1,26)=0.86$, $p=0.36$; target: $F(1,26)=0.29, p=0.59)$.

\section{Psychometric analysis of sequential effects}

Rather than examining the sequential effects only with the highly recognizable targets, we included more stimuli along the continua to perform additional psychometric analysis. For this analysis, we calculated the proportions of current responses that agreed with the immediately preceding responses (i.e., the proportions of the same category choices) for all highly recognizable current faces (P-, M-faces, and targets) in each identity category as well as for ambiguous current faces located at the category boundary according to each preceding stimulus type. In other words, we analyzed the modal responses to current faces when preceding and current faces originated from the same category and the nonmodal responses when the two faces originated from different categories. Responses to the current faces were then pooled together, depending on whether the preceding and current faces originated from the same or different categories. Next, we fit the data along the continua using a sigmoidal function based on 
A Male participant -Male target

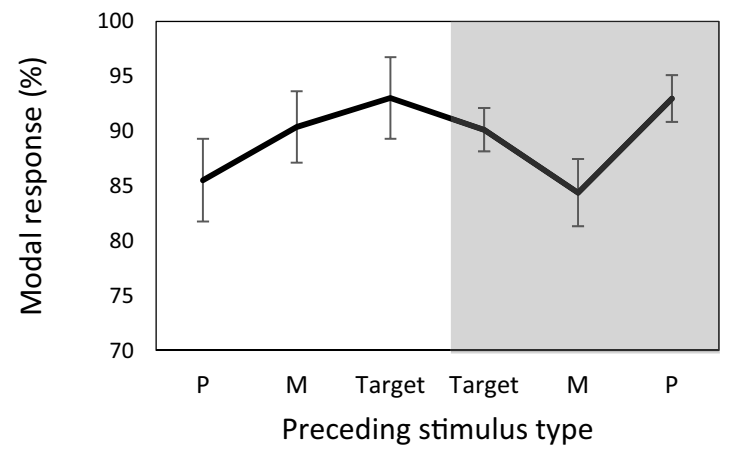

C Female participant -Male target

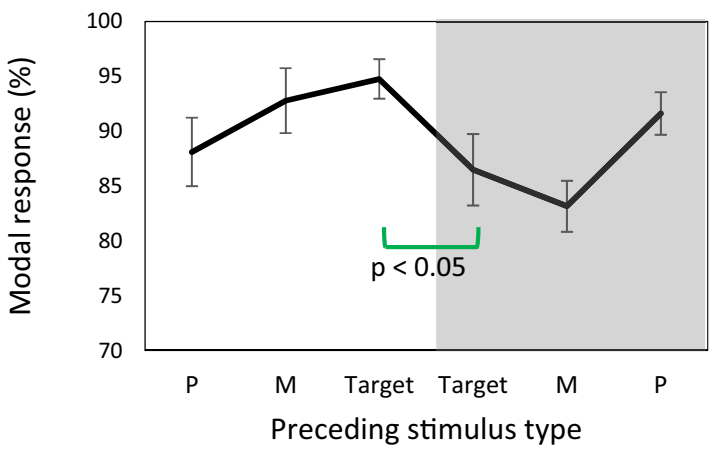

Fig. 3 Modal responses of the current facial targets as a function of the preceding stimuli presented two trials back when male participants categorized male (a) and female (b) targets and female participants categorized male (c) and female (d) targets. The gray zones indicate that the preceding and current facial stimuli have different category

facial and participant genders (Thielscher \& Pessoa, 2007). The $50 \%$ points and the slopes of the fitted curves served as the indices for comparison across preceding stimulus conditions.

As shown in Fig. 5, the psychometric curves, particularly for the current faces in the middle region around the category boundaries, were shifted from right to left with increasing relative distance between the preceding and current stimuli. Trend analyses confirmed this visual inspection. While the slopes remained comparable across preceding stimulus conditions (all $p s>0.172$ ), the $50 \%$ points of the psychometric functions significantly varied in a linear manner when male participants categorized both male $(F(1,13)=29.84$, $p<0.001$, Fig. 5a) and female faces $(F(1,13)=29.87$, $p<0.001$, Fig. $5 \mathrm{~b})$ and when female participants categorized both male $(F(1,13)=16.23, p=0.001$, Fig. $5 \mathrm{c})$ and female faces $(F(1,13)=13.45, p=0.003$, Fig. $5 \mathrm{~d})$. This direction of the shifts is closely in line with our previous results, which show that during the same-category/different-category transitions, an increased interstimulus distance is accompanied by reduced/enhanced modal responses for the current targets. This is because those relative-distance effects equivalently
B

Male participant -Female target

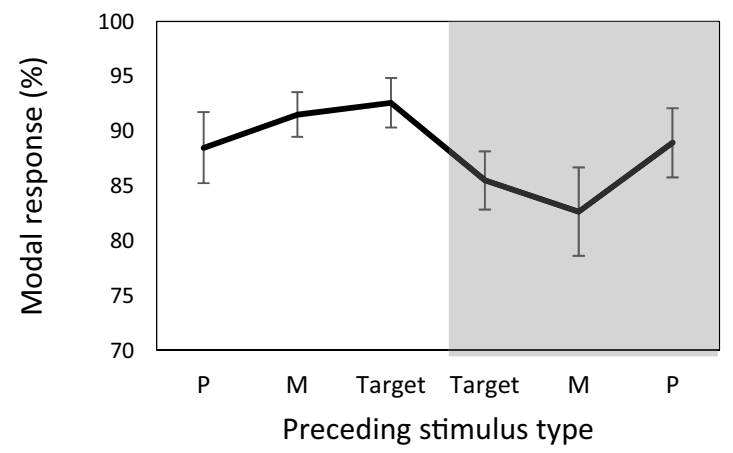

D Female participant -Female target

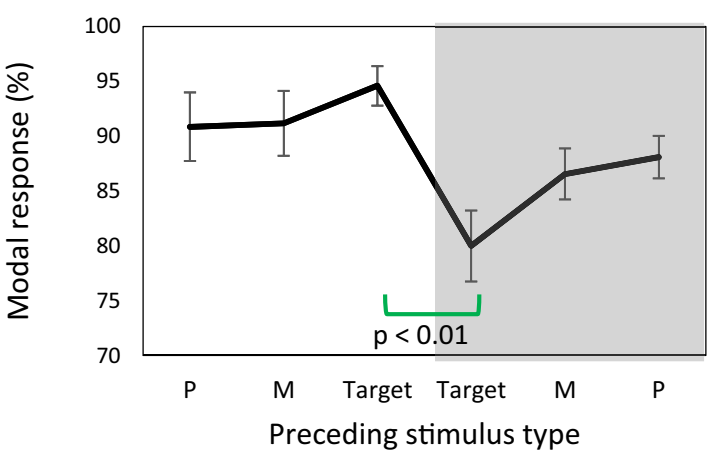

memberships. Error bars represent \pm SEM. After being collapsed across facial and participant genders, the average number of trials used to compute the data for each preceding stimulus type was as follows. P, $9.82 \pm 0.91 ; \mathrm{M}, 8.86 \pm 0.82$; Target, $7.77 \pm 0.67$; Target (gray zone), $8.71 \pm 0.90 ; \mathrm{M}$ (gray zone), $8.95 \pm 0.86 ; \mathrm{P}$ (gray zone), $9.09 \pm 0.79$

indicate that the proportion of current responses that agree with preceding responses is decreased with increasing interstimulus distance.

When there was a two-trial gap between the preceding and current stimuli (Fig. 6), the $50 \%$ points of the curves also shifted linearly from right to left with increasing relative distance between the preceding and current stimuli. This pattern of results was observed for both male and female participants when they categorized either male or female faces (all $p s \leq$ $0.05)$. The slopes of the curves did not significantly differ (all $p s>0.75$ ). However, these findings are inconsistent with the results in which the targets were analyzed along (see the "Long-term sequential effects" section). We reason that this discrepancy could be attributed to the fact that in the psychometric analyses, sequential effects were accumulated across all data points and ambiguous current faces also were included. Indeed, for the ambiguous current faces, categorization responses varied linearly with increasing interstimulus distance, regardless of whether the faces were preceded by the immediately preceding face (Fig. 7, all $p s<0.004$ ) or the preceding face presented two trials back (Fig. 8, all $p s \leq$ 0.05 , except when female participants judged female faces: 
Fig. 4 Categorization data of selected P-faces (a), M-faces (b) and targets (c) as a function of facial and participant gender. Error bars represent \pm SEM

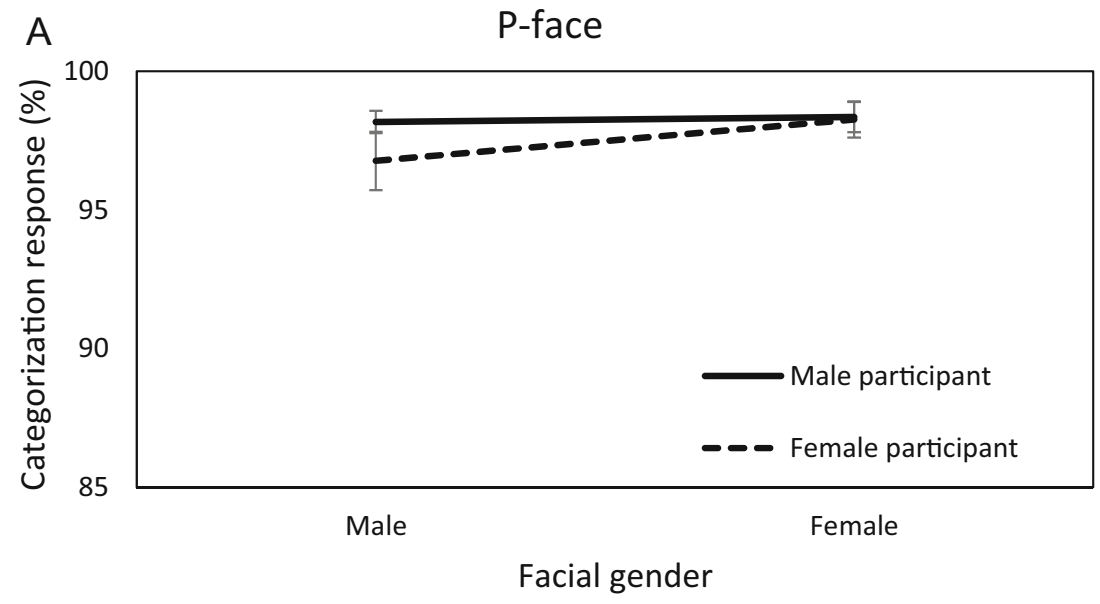

B

M-face

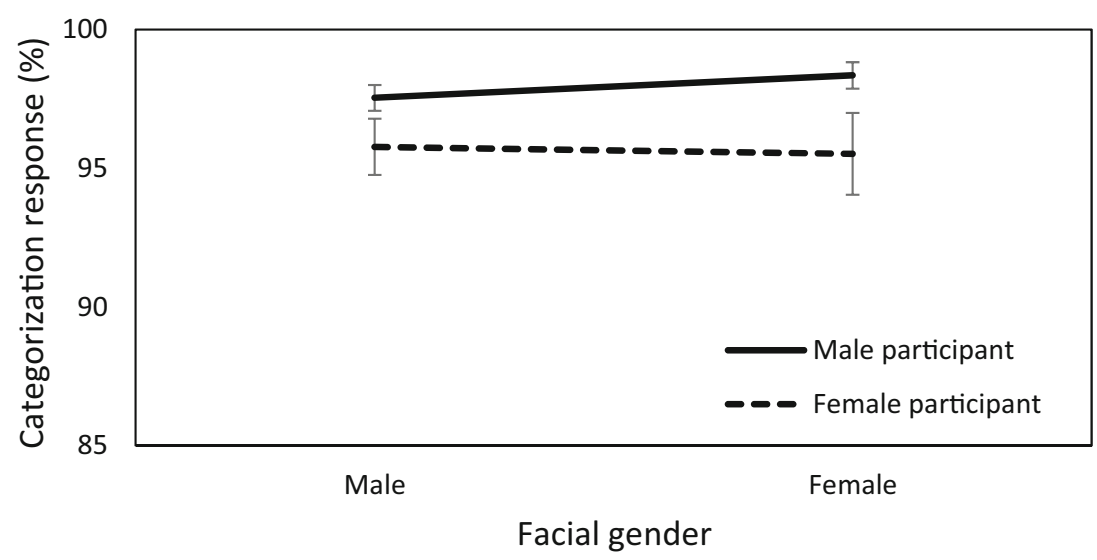

C

Target face

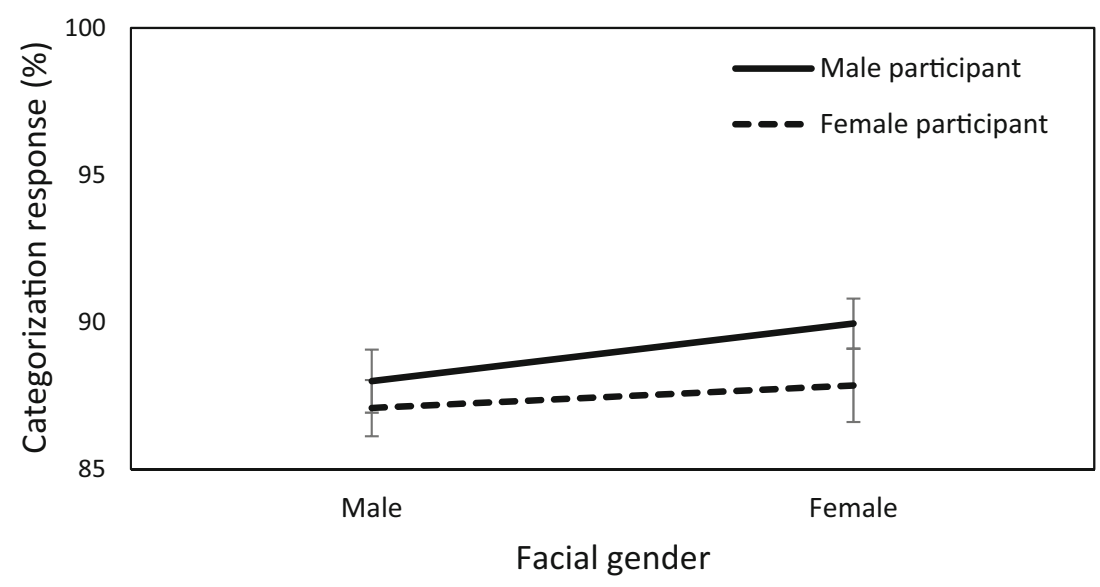

$F(1,13)=2.24, p=0.15)$. Notably, because the ambiguous faces do not belong to any identity category and have a categorization rate approximately $50 \%$, we calculated the proportion of choices assigned to a distinct identity category instead of modal responses. For the same reason, we did not examine the presence of assimilation or contrast effects for the ambiguous faces.

\section{Discussion}

\section{Role of relative information in identity-based sequential effects}

When categorizing the identities of a sequence of randomly presented faces, categorization performance on current targets 

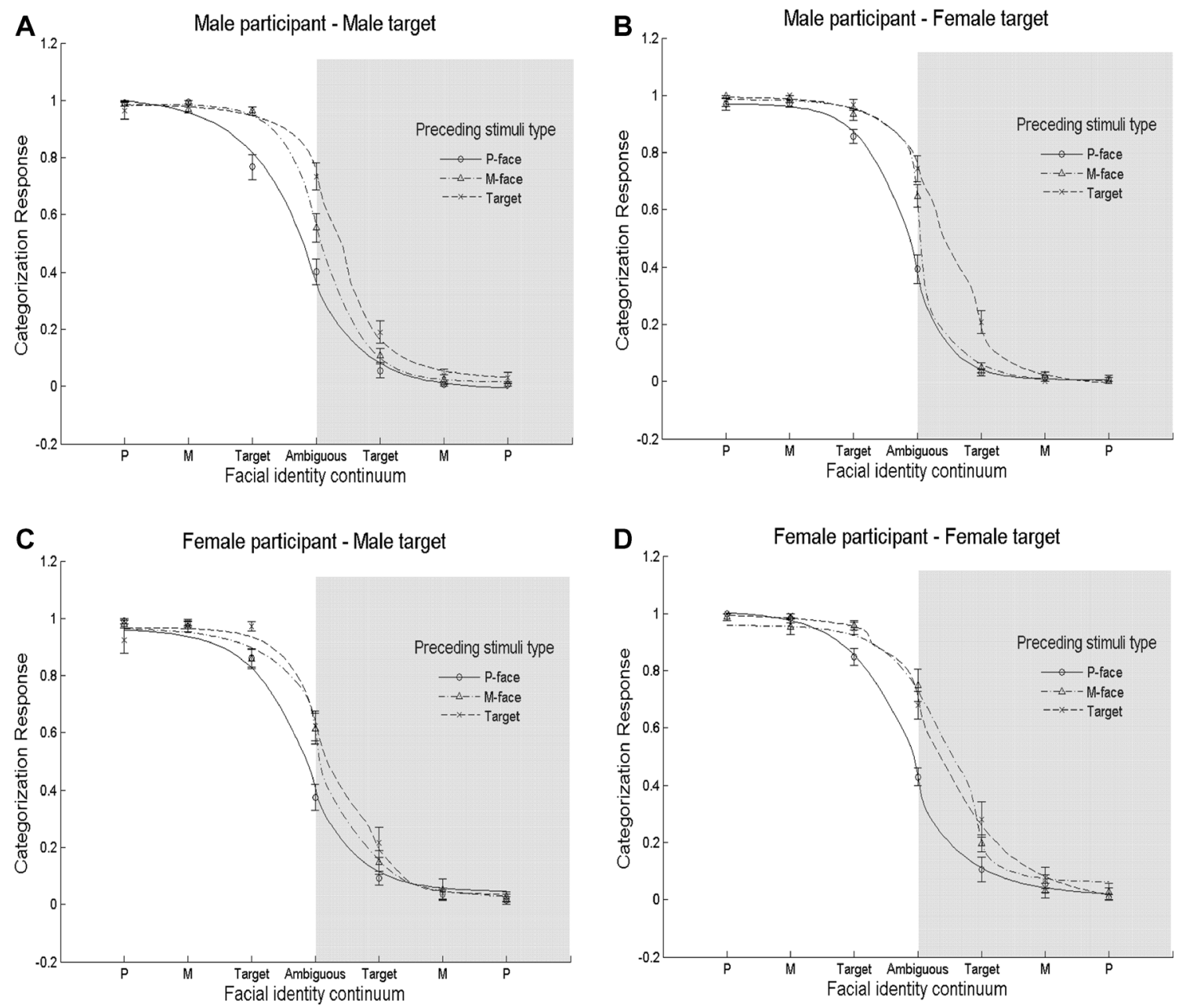

Fig. 5 Psychometric data of the facial-identity continuum as a function of the preceding stimuli when male participants categorized male (a) and female (b) continua and female participants categorized male (c) and female (d) continua. The y-axis represents the proportions of current responses that agreed with the immediately preceding responses. The $\mathrm{x}$ axis represents the current faces along the continuum, including the selected faces depicted in Fig. 1a and the ambiguous faces located at the category boundary. The curves denote the fitted psychometric

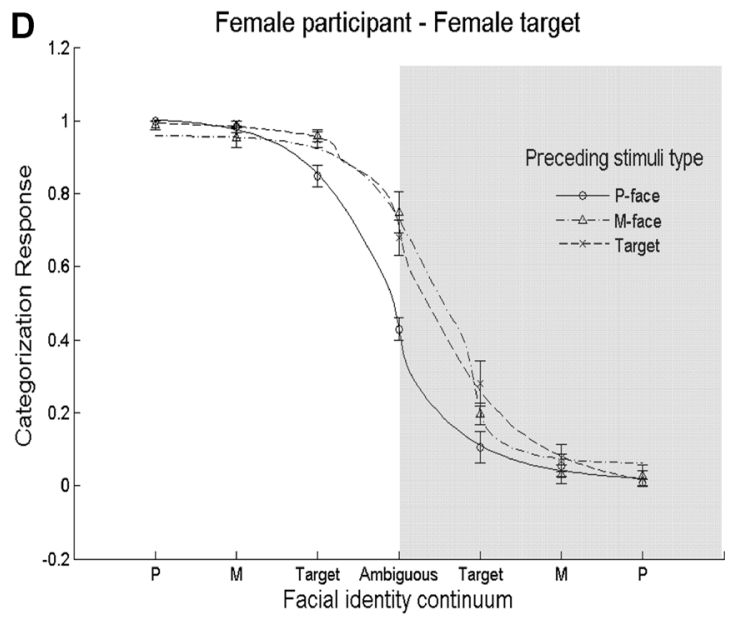

functions, whereas the dots denote the original data. The gray zones indicate that the preceding and current facial stimuli have different category memberships. Error bars represent $\pm S E M$. After being collapsed across the preceding stimulus types and facial and participant genders, the average number of trials used to compute the data for each preceding stimulus type are as follows. P, $8.62 \pm 0.80 ; \mathrm{M}, 8.58 \pm 0.79$; Target, $9.15 \pm 0.82$; Ambiguous face, $9.39 \pm 0.75$; Target (gray zone), $8.96 \pm 0.00 ; \mathrm{M}$ (gray zone), $8.65 \pm 0.72 ; \mathrm{P}$ (gray zone), $8.83 \pm 0.86$

was influenced by the local sequential context provided by the immediately preceding faces. This influence also was observed to some extent when there was a two-trial gap between the preceding and current faces. Furthermore, consistent with previous research (Hampton, et al., 2005; Stewart \& Brown, 2005), these identity-based sequential effects depend on the relative distance between the preceding and current stimuli, which suggests that the local sequential context is used to inform the judgments of facial identity. Accordingly, the present study reveals that in addition to the information conveyed by the faces, facial identity is judged according to preceding contextual information.

However, alternative explanations need to be ruled out. First, could the observed results reflect a consequence of well-established facial identity aftereffects (Leopold, O'Toole,

Vetter, \& Blanz, 2001), because the identity aftereffects also involve a biased recognition of current facial identity after a period of stimulation from preceding faces? However, unlike the experimental procedure adopted in this study, a prolonged adaptation to preceding faces of more than 1 second is typically required to generate robust aftereffects (Strobach \& Carbon, 2013). In addition to the identity aftereffects, response bias, in which participants are biased against/toward making two identical responses in a row, is incompatible with the present results. In our analysis, we excluded the trials in which the targets were preceded by "inaccurate" preceding stimuli. In other words, the preceding responses were all identical (i.e., they were all "accurate") regardless of preceding stimulus types during either the same-category or different-category transitions. Therefore, if there exists response bias, 

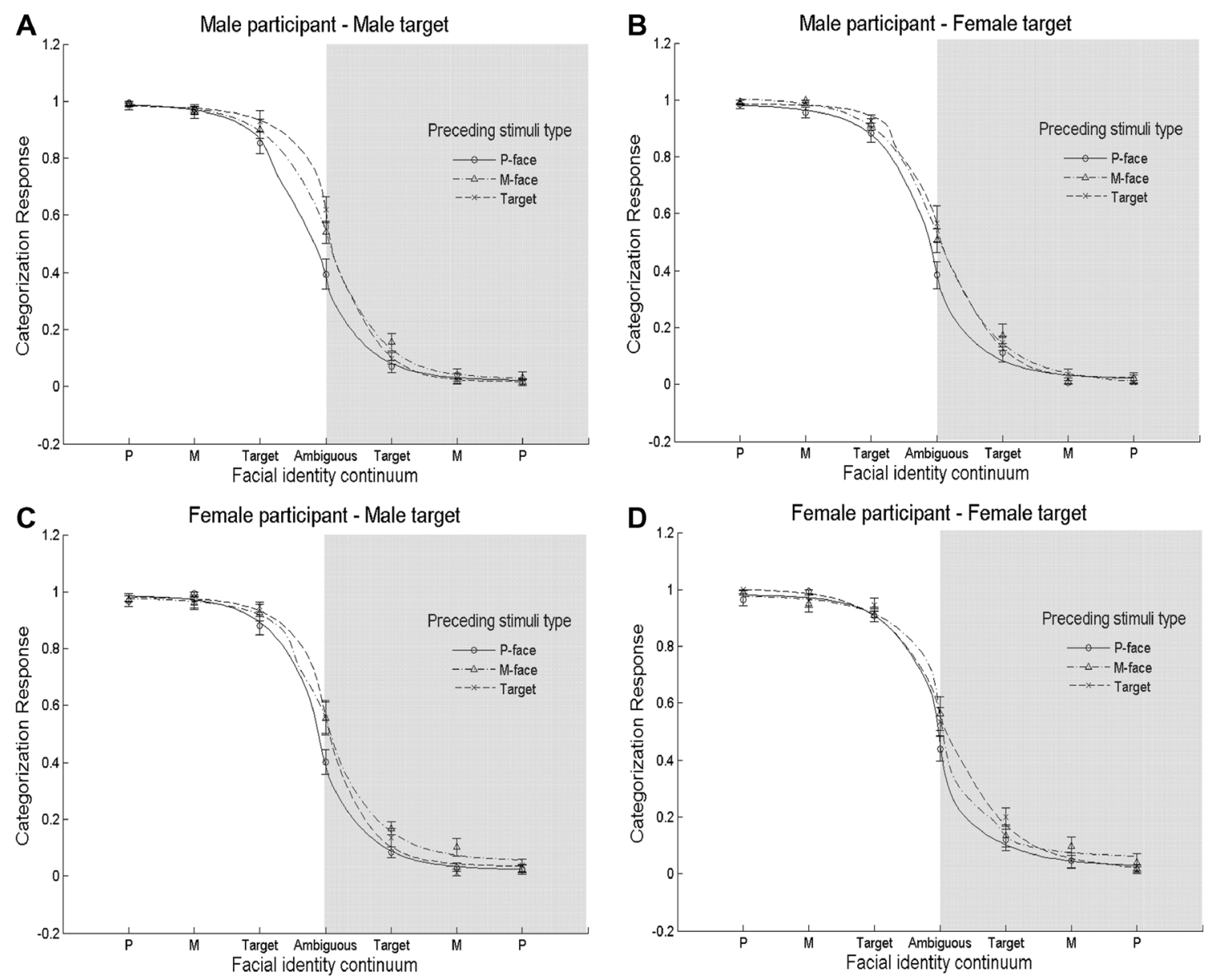

Fig. 6 Psychometric data of the facial-identity continuum as a function of the preceding stimuli presented two trial back when male participants categorized male (a) and female (b) continua and female participants categorized male (c) and female (d) continua. The y-axis represents the proportions of current responses that agreed with the immediately preceding responses. The x-axis represents the selected current faces depicted in Fig. 1a and the ambiguous faces located at the category boundary. The curves denote the fitted psychometric functions, whereas

categorization performance should be comparable across preceding contexts during either category transition. However, this prediction clearly contradicts the reported relativedistance effects. Is it possible that our results may be related to facial-identity priming effects (Schweinberger, Pickering, Jentzsch, Burton, \& Kaufmann, 2002), in which performance is improved when stimuli are preceded by highly similar faces (e.g., repetition priming)? However, there are some important differences between our results and facial-identity priming effects. First, priming effects generally affect reaction time (RT), whereas our findings are indexed by categorization rates. Second, during the different-category transitions, performance was actually decreased following more similar preceding faces. To further address the potential involvement of repetition priming, we conducted additional analyses to investigate whether RTs were gradually shorter with decreasing relative distance between the preceding and current stimuli the dots denote the original data. The gray zones indicate that the preceding and current facial stimuli have different category memberships. Error bars represent \pm SEM. After being collapsed across preceding stimulus types and facial and participant genders, the average number of trials used to compute the data for each preceding stimulus type are as follows. P, $8.99 \pm 0.80 ; \mathrm{M}, 8.72 \pm 0.75$; Target, $8.82 \pm 0.83$; Ambiguous face, $9.02 \pm 0.74$; Target (gray zone), $8.92 \pm 0.85$; M (gray zone), $8.98 \pm 0.87 ; \mathrm{P}$ (gray zone), $8.63 \pm 0.78$

during the same-category transitions, such that RTs were the shortest when the targets were preceded by themselves. Our analyses did not support this prediction, as RTs were comparable across preceding stimulus conditions in most conditions (trend analysis, all $p s>0.07$ except when female participants judged female faces, $F(1,13)=12.92, p=0.003)$. Furthermore, could the assimilation effects reflect categorical priming (Wiese \& Schweinberger, 2008), in which performance of current faces is improved following the faces from the same category relative to the faces from the opposite category? For the assimilation effects observed in both male and female participants, we did observe reduced RTs when the current targets were preceded by the targets themselves compared with when preceded by the targets from the opposite category (paired $t$-test, all $p s<0.05$ ). However, reduced RTs also were observed in the contrast effects, as the RTs for the current targets were shorter following the P-faces from the same 
A Male participant -Male target

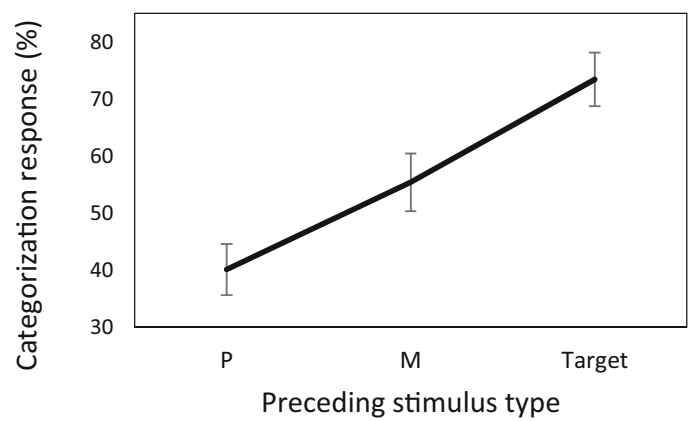

C Female participant -Male target

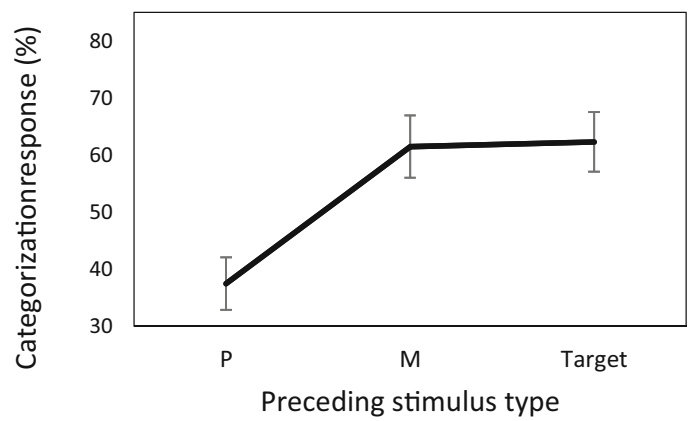

Fig. 7 Categorization responses of the current ambiguous faces as a function of the preceding stimuli when male participants categorized male (a) and female (b) faces and female participants categorized male (c) and female (d) faces. The gray zones indicate that the preceding and current facial stimuli have different category memberships. Error bars

category compared with the P-faces from the opposite category (all $p s<0.05$ except when male participants judged male faces). Thus, we suggest that categorical priming effects cannot fully explain the assimilation effects. Further investigation is necessary to better determine the relationship between priming and sequential effects.

\section{Distinct nature of sequential effects between male and female participants}

Although identity-based sequential effects were observed in both male and female participants, the nature of these effects appeared to be qualitatively different. For male participants, sequential effects involved contrast bias when the interstimulus distance was large and assimilation bias when the interstimulus distance was small. In contrast, for female participants, sequential effects involved only assimilation bias when the distance was small and these effects could still be observed when a two-trial gap was included between the preceding and current stimuli.

To account for assimilation/contrast effects during categorization, the similarity-dissimilarity generalized context model (Stewart \& Brown, 2005; Stewart et al., 2002)
B Male participant -Female target

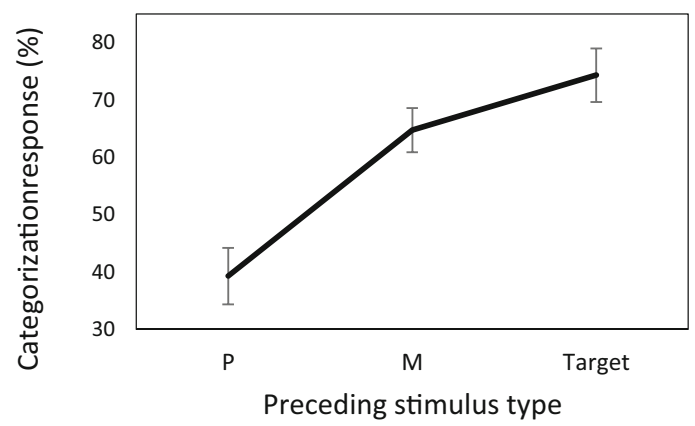

D Female participant -Female target

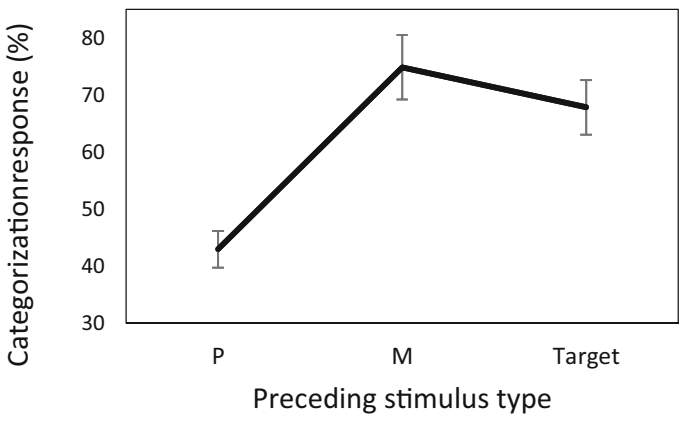

represent \pm SEM. After being collapsed across facial and participant genders, the average number of trials used to compute the data for each preceding stimulus type are as follows. P, $10.16 \pm 0.81$;, $9.07 \pm 0.69$; Target, $8.95 \pm 0.71$

suggests that the effects reflect the result of a particular decision strategy in which relative difference information between successive items is used by participants to inform their categorization decisions. In this view, when the relative distance between two successive faces is increasingly large, participants might believe that the two faces are dissimilar. As a result, participants would tend to judge the preceding and current faces as belonging to different categories (contrast effect). Conversely, when the relative distance between the two successive faces is increasingly small, participants might believe that the two faces are similar. As a result, participants would tend to judge the current face as belonging to the same category as the preceding face (assimilation effects).

Echoing this view, a model of social judgment (Mussweiler, 2003) contends that the consequence of relative judgment (i.e., contrast or assimilation) depends on whether participants focus on the similarity or dissimilarity between the preceding and current stimuli. One study (Damisch, Mussweiler, \& Plessner, 2006) showed that when facing a sequence of performances in the gymnastics competition in the 2004 Olympic Games, participants tended to assimilate their evaluations of the current performance toward the 
A Male participant -Male target

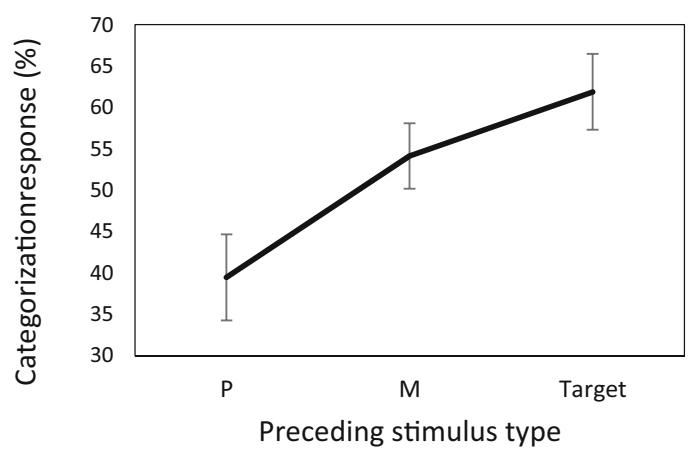

C Female participant -Male target

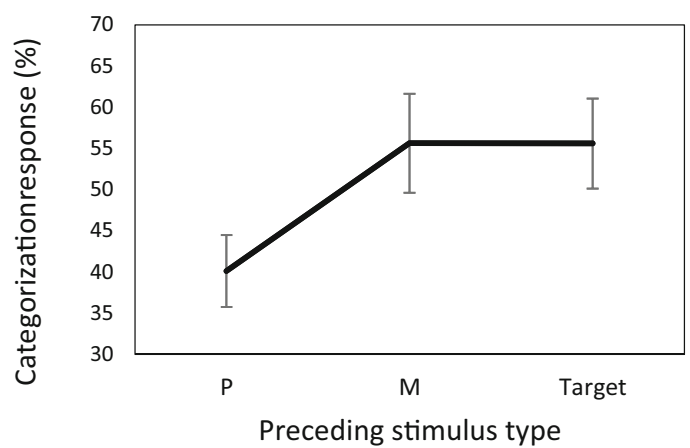

Fig. 8 Categorization responses of the current ambiguous faces as a function of the preceding stimuli presented two trials back when male participants categorized male (a) and female (b) faces and female participants categorized male (c) and female (d) faces. The gray zones indicate that the preceding and current facial stimuli have different

judgments of the preceding performance if participants were induced to focus on the similarities between the successive performances. Conversely, a focus on dissimilarities led participants to contrast their judgments away from the preceding performance. Based on a foundation of previous research, the present study suggests that, when making perceptual judgments on a sequence of facial identities, female participants may focus on the similarities between the preceding and current faces and thereby produce assimilation effects; however, male participants may focus on both inter-stimulus similarities and dissimilarities, which results in corresponding assimilation and contrast effects.

\section{Conclusions}

Although sequential effects in perceptual judgment have long been established, the issue has been largely ignored in the facial identity perception literature. One possible reason is that whether relative information between preceding and current stimuli is used in facial identity perception is extraneous to the theory pursued. Therefore, sequential effects often are treated as noise in the majority of previous
B Male participant -Female target

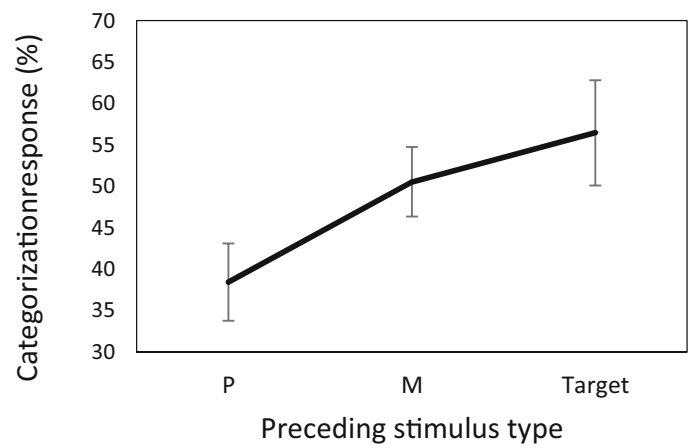

D Female participant -Female target

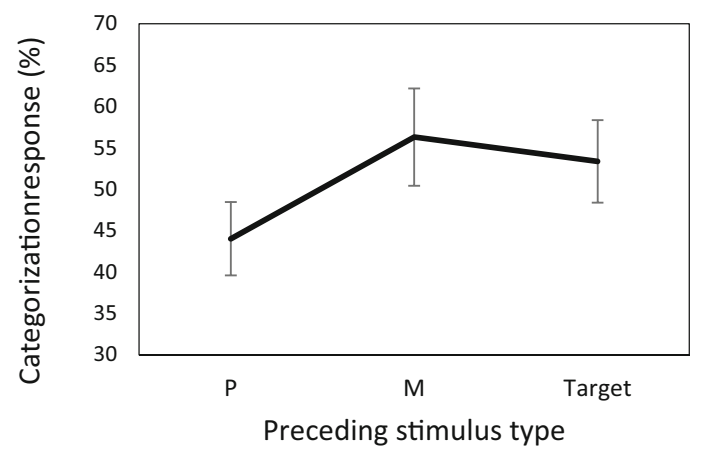

category memberships. Error bars represent \pm SEM. After being collapsed across facial and participant genders, the average number of trials used to compute the data for each preceding stimulus type are as follows. P, $9.64 \pm 0.76$; M, $9.02 \pm 0.83$; Target, $8.39 \pm 0.58$

studies and can be ignored by averaging the responses to randomly presented facial stimuli over trials. The present study demonstrates that the application of sequential effects can provide important insights into the nature of facial identity perception. We suggest that facial identity perception is not unitary; instead, it is a multifaceted process. First, in addition to the information values conveyed by faces, the relative information regarding current faces and preceding contexts also plays a contributing role in perceptual judgments. Second, when relative information is used as evidence to inform judgment, females tend to assimilate their judgments toward the category of the preceding faces whereas males tend to either assimilate or contrast their judgments toward or away from the category of the preceding faces, depending on the relationship between preceding and current faces. Recent studies also have reported sequential effects in the perceptual judgments of other facial domains, such as face perception (Liberman, Fischer, \& Whitney, 2014), facial expression (Hsu \& Yang, 2013) and facial attractiveness (Kondo, Takahashi, \& Watanabe, 2012, 2013; Kramer, Jones, \& Sharma, 2013). With the present study, these findings suggest the inherent nature of relative judgment in face perception in general. 
Acknowledgements This work was supported by the National Science Council of Taiwan, R.O.C. (NSC 102-2410-H-004-045-MY2). The authors thank Chi-Tai Huang for helpful suggestion during the preparation of the manuscript, Lee-Xieng Yang for psychometric analysis, and Paul Chang for technical assistance.

\section{References}

Bruce, V., \& Young, A. (1986). Understanding face recognition. British Journal Psychology, 77, 305-327.

Damisch, L., Mussweiler, T., \& Plessner, H. (2006). Olympic medals as fruits of comparison? Assimilation and contrast in sequential performance judgements. Journal of Experimental Psychology: Applied, $12,166-178$

Ganel, T., \& Goshen-Gottstein, Y. (2002). Perceptual integrality of sex and identity of faces: Further evidence for the single-route hypothesis. Journal of Experimental Psychology: Human Perception and Performance, 28, 854-867.

Hampton, J. A., Estes, Z., \& Simmons, C. L. (2005). Comparison and contrast in perceptual categorization. Journal of Experimental Psychology: Learning, Memory, and Cognition, 31, 1459-1476.

Helson, H. (1964). Current trends and issues in adaptation-level theory. American Psychologist, 19, 26-38.

Herlitz, A., \& Loven, J. (2013). Sex differences and the own-gender bias in face recognition: A meta-analysis review. Visual Cognition, 21, 1306-1336.

Hsu, S. M., \& Yang, L. X. (2013). Sequential effects in facial expression categorization. Emotion, 13, 573-586.

Kondo, A., Takahashi, K., \& Watanabe, K. (2012). Sequential effects in face-attractiveness judgment. Perception, 41, 43-49.

Kondo, A., Takahashi, K., \& Watanabe, K. (2013). Influence of gender membership on sequential decisions of face attractiveness. Attention, Perception, \& Psychophysics, 75, 1347-1352.

Kramer, R. S. S., Jones, A. L., \& Sharma, D. (2013). Sequential effects in judgements of attractiveness: The influences of face race and sex. Plos One, 8, e82226.

Laming, D. R. J. (1997). The measurement of sensation. Oxford: Oxford University Press.

Leopold, D. A., O'Toole, A. J., Vetter, T., \& Blanz, V. (2001). Prototypereferenced shape encoding revealed by high-level aftereffects. Nature Neuroscience, 4, 89-94.

Lewin, C., \& Herlitz, A. (2002). Sex differences in face recognition Women's faces make the difference. Brain and Cognition, 50, $121-128$.
Liberman, A., Fischer, J., \& Whitney, D. (2014). Serial dependence in the perception of faces. Current Biology, 24, 2569-2574.

Lockhead, G. R. (2004). Absolute judgments are relative: A reinterpretation of some psychophysical ideas. Review of General Psychology, $8,265-272$.

Mussweiler, T. (2003). Comparison processes in social judgment: Mechanisms and consequences. Psychological Review, 110, 472489.

Rhodes, G., Jaquet, E., Jeffery, L., Evangelista, E., Keane, J., \& Calder, A. J. (2011). Sex-specific norms code face identity. Journal of Vision, 11, doi:10.1167/11.1.1

Schweinberger, S. R., Pickering, E. C., Jentzsch, I., Burton, A. M., \& Kaufmann, J. M. (2002). Event-related brain potential evidence for a response of inferior temporal cortex to familiar face repetitions. Cognitive Brain Research, 14, 398-409.

Stewart, N., \& Brown, G. D. A. (2005). Similarity and dissimilarity as evidence in perceptual categorization. Journal of Mathematical Psychology, 49, 403-409.

Stewart, N., Brown, G. D. A., \& Chater, N. (2002). Sequential effects in categorization of simple perceptual stimuli. Journal of Experimental Psychology: Learning, Memory, and Cognition, 28, 3-11.

Strobach, T., \& Carbon, C. C. (2013). Face adaptation effects: Reviewing the impact of adapting information, time, and transfer. Frontiers in Psychology, 4, doi:10.3389/fpsyg.2013.00318

Thielscher, A., \& Pessoa, L. (2007). Neural correlates of perceptual choice and decision making during fear-disgust discrimination. Journal Neuroscience, 27, 2908-2917.

Valentine, T. (1991). A unified account of the effects of distinctiveness, inversion, and race in face recognition. The Quarterly Journal of Experimental Psychology A: Human Experimental Psychology, 43, 161-204.

Ward, L. M., \& Lockhead, G. R. (1970). Sequential effects and memory in category judgments. Journal of Experimental Psychology, 84, 27-34.

Wiese, H., \& Schweinberger, S. R. (2008). Event-related potentials indicate different processes to mediate categorical and associative priming in person recognition. Journal of Experimental PsychologyLearning Memory and Cognition, 34, 1246-1263.

Willenbockel, V., Sadr, J., Fiset, D., Horne, G. O., Gosselin, F., \& Tanaka, J. W. (2010). Controlling low-level image properties: The SHINE toolbox. Behavior Research Methods, 42, 671-684.

Wright, D. B., \& Sladden, B. (2003). An own gender bias and the importance of hair in face recognition. Acta Psychologica, 114, 101-114.

Zotov, V., Jones, M. N., \& Mewhort, D. J. K. (2011). Contrast and assimilation in categorization and exemplar production. Attention, Perception, \& Psychophysics, 73, 621-639. 